\title{
Aspirin-triggered resolvin D1 reduces mucosal inflammation and promotes resolution in a murine model of acute lung injury
}

\author{
O Eickmeier ${ }^{1}$, H Seki ${ }^{1}$, O Haworth ${ }^{1}$, JN Hilberath ${ }^{1}$, F Gao ${ }^{2}$, M Uddin ${ }^{1}$, RH Croze ${ }^{1}$, T Carlo ${ }^{1}$, \\ MA Pfeffer ${ }^{1}$ and BD Levy ${ }^{1,2}$
}

Acute lung injury (ALI) is a severe illness with excess mortality and no specific therapy. Protective actions were recently uncovered for docosahexaenoic acid-derived mediators, including D-series resolvins. Here, we used a murine selflimited model of hydrochloric acid-induced ALI to determine the effects of aspirin-triggered resolvin D1 (AT-RvD1; $7 S, 8 R, 17 R$-trihydroxy-4Z,9E,11E,13Z,15E,19Z-docosahexaenoic acid) on mucosal injury. RvD1 and its receptor ALX/FPR2 were identified in murine lung after ALI. AT-RvD1 $\left(\sim 0.5-5 \mu \mathrm{g} \mathrm{gg}^{-1}\right)$ decreased peak inflammation, including bronchoalveolar lavage fluid (BALF) neutrophils by $\sim 75 \%$. Animals treated with AT-RvD1 had improved epithelial and endothelial barrier integrity and decreased airway resistance concomitant with increased BALF epinephrine levels. AT-RvD1 inhibited neutrophil-platelet heterotypic interactions by downregulating both P-selectin and its ligand CD24. AT-RvD1 also significantly decreased levels of BALF pro-inflammatory cytokines, including interleukin (IL)-1 $\beta$, IL-6, Kupffer cells, and tumor necrosis factor- $\alpha$, and decreased nuclear factor- $\kappa \mathrm{B}-$ phosphorylated p 65 nuclear translocation. Taken together, these findings indicate that AT-RvD1 displays potent mucosal protection and promotes catabasis after ALI.

\section{INTRODUCTION}

Acute lung injury (ALI) and the acute respiratory distress syndrome (ARDS) are life-threatening disorders that contribute significantly to critical illness. ${ }^{1}$ Approximately 75,000 patients die from ARDS each year in the United States. ${ }^{2}$ Respiratory failure from ALI or ARDS is a frequent cause of admission to intensive care units, often requiring ventilatory support. Despite advances in understanding ARDS pathophysiology, no medical interventions have proven effective in improving the outcome of patients with ALI/ARDS. ${ }^{3,4}$ Aspiration pneumonitis is one of the leading causes of ALI/ARDS that in most instances is self-limited, ${ }^{5}$ suggesting the existence of endogenous, host-protective mechanisms.

Although the cellular events that return the injured lung to homeostasis have been described by histopathology, there remains only a limited understanding of the molecular mechanisms underlying this catabatic process. Polyunsaturated fatty acids appear to play important roles in ALI and its resolution. Activation of pulmonary leukocytes leads to increased generation of arachidonic acid-derived eicosanoids that can serve as important mediators of inflammation, vascular leak, and tissue catabasis. ${ }^{6-8}$ In contrast, $\omega$-3 fatty acid-based nutritional supplementation can significantly improve oxygenation and decrease the length of stay in an intensive care unit, time on mechanical ventilation, occurrence of new organ failure, and mortality. ${ }^{9-11}$

Airway mucosa is enriched with the $\omega-3$ fatty acid docosahexaenoic acid (DHA). ${ }^{12}$ Resolvin D1 (RvD1) is a DHA-derived mediator first identified in resolving peritoneal exudates. ${ }^{13}$ Aspirin acetylates cyclooxygenase- 2 to lead to the formation of aspirin-triggered mediators, including the $17 R$-epimer of RvD1 termed aspirin-triggered RvD1 (AT-RvD1; 7S,8R,17Rtrihydroxy-4Z,9E,11E,13Z,15E,19Z-docosahexaenoic acid) ${ }^{13,14}$ that decreases leukocytic infiltration in murine peritonitis to a greater extent than RvD1 and is more resistant to catalysis than RvD1. ${ }^{14}$ Of interest, both cyclooxygenase- 2 and aspirin may have beneficial actions in ALI/ARDS. ${ }^{15,16}$ Here, in a non-lethal model of ALI, AT-RvD1 decreased lung inflammation after mucosal

\footnotetext{
${ }^{1}$ Pulmonary and Critical Care Medicine, Department of Internal Medicine, Brigham and Women's Hospital and Harvard Medical School, Boston, Massachusetts, USA. ${ }^{2}$ Center for Experimental Therapeutics and Reperfusion Injury, Department of Anesthesiology, Perioperative and Pain Medicine, Brigham and Women's Hospital and Harvard Medical School, Boston, Massachusetts, USA. Correspondence: BD Levy (blevy@partners.org) 
injury and promoted ALI resolution by enhancing restitution of barrier integrity, decreasing circulating neutrophil-platelet heterotypic interactions, and regulating inflammatory mediators and nuclear factor- $\mathrm{\kappa B}(\mathrm{NF}-\kappa \mathrm{B})$ activation.

\section{RESULTS}

\section{RvD1 and 17(S)-hydroxy-DHA are generated after} mucosal injury

To determine if endogenous DHA is converted to RvD1 after ALI, murine lungs were obtained $12 \mathrm{~h}$ after ALI from intratracheal $\mathrm{HCl}(0.1 \mathrm{~N}, \mathrm{pH} 1.5)$ and lipids were extracted from lung homogenates for analyses (see Methods). Both RvD1 and its biosynthetic precursor 17(S)-hydroxy-DHA were identified by liquid chromatography with tandem mass spectrometry-based metabololipidomics analysis (Figure 1a-c). At this time point, RvD1 was present in pg quantities.

\section{ALX/FPR2 receptors are expressed on lung epithelial cells and macrophages}

RvD1 and AT-RvD1 are anti-inflammatory and pro-resolving agonists at ALX/FPR2 receptors; ${ }^{17,18}$ thus, the expression of ALX/FPR2 was determined by immunohistochemistry in murine lung at baseline and after ALI (Figure 1d-g). ALX/FPR2 receptors were expressed at low levels in uninjured lungs, in particular in airway epithelial cells and alveolar macrophages (MACs) (Figure 1d and e). As early as $2 \mathrm{~h}$ after ALI, there was an evident increase in mucosal epithelial cell ALX/FPR2 expression (Figure 1f) that was further increased $12 \mathrm{~h}$ after ALI (Figure 1g).

\section{AT-RvD1 reduces leukocyte recruitment after airway mucosal injury}

With both RvD1 and its receptor ALX/FPR2 present in murine lung, we determined the impact of exogenous AT-RvD1 on inflammatory responses after ALI. Because RvD1 is rapidly inactivated, ${ }^{14}$ its epimer AT-RvD1, which partially resists metabolic inactivation, was chosen for study. Like RvD1, AT-RvD1 is an agonist at ALX/FPR2 receptors. ${ }^{18}$ In a first series of experiments, AT-RvD1 or a vehicle control $(0.1 \%$ ethanol (vol/vol)) were administered intravenously $15 \mathrm{~min}$ before instillation of $\mathrm{HCl}(0.1 \mathrm{~N}, \mathrm{pH} 1.5)$ into the left mainstem bronchus (Figure 2a). AT-RvD1 led to significant and dosedependent decrements in total bronchoalveolar lavage fluid (BALF) cell numbers $12 \mathrm{~h}$ after ALI $(67,500 \pm 15,880$ total BALF cells (AT-RvD1 $10 \mathrm{ng}, n=5) ; 53,810 \pm 7,836$ total BALF cells (AT-RvD1 $100 \mathrm{ng}, n=10$ ); mean \pm s.e.m.) relative to vehicle control mice $(129,800 \pm 10,230$ total BALF cells; mean \pm s.e.m., $n=12, P<0.01$ ) (Figure 2b). In particular, AT-RvD1 decreased BALF neutrophils (polymorphonuclear leukocytes (PMNs)) $(11,550 \pm 4,841$ PMNs (AT-RvD1 $10 \mathrm{ng}, n=5) ; 8,258 \pm 2,988$ PMNs (AT-RvD1 $100 \mathrm{ng}, n=10)$; mean \pm s.e.m.) relative to vehicle $(36,650 \pm 5,649$ PMNs $(n=12)$; mean \pm s.e.m., $P<0.01)$ (Figure 2c). BALF MACs were also decreased with AT-RvD1 $(44,470 \pm 7,846$ MACs (AT-RvD1 100 ng, $n=10)$; mean \pm s.e.m.) in comparison to vehicle $(91,130 \pm 10,890$ MACs; mean \pm s.e.m., $n=12, P<0.01)$ (Figure 2d).
In a second series of experiments to determine its proresolving and potential therapeutic properties, AT-RvD1 was given $2 \mathrm{~h}$ after airway injury (Figure 2e). When administered post-injury, AT-RvD1 also mediated significant decreases in total BALF cells $(87,860 \pm 14,550$ (100 ng, $n=7$ ); 130,000 $\pm 7,935$ (vehicle, $n=6$ ); mean \pm s.e.m., $P<0.05$ ) (Figure 2f) and BALF PMNs (10,520 1,404 (100 ng, $n=7)$; $33,410 \pm 4,885$ (vehicle, $n=6$ ); mean \pm s.e.m., $P<0.01$ ) (Figure 2g). No significant changes in the numbers of BALF MACs were present when AT-RVD1 (100 ng) was given after injury (Figure 2h).

In a third series of experiments to investigate AT-RvD1's impact on early leukocyte trafficking after ALI, airway inflammation was monitored by BAL 2 and $6 \mathrm{~h}$ after ALI. Although no significant differences in the numbers of total BALF cells were identified between AT-RvD1 dosing before $(-15 \mathrm{~min})$ or after $(+2 \mathrm{~h})$ ALI, there was a lower amplitude and earlier decrease in airway inflammation in mice that received AT-RvD1 before ALI. These changes in early BALF cell numbers were correlated with BALF MACs, as significant numbers of BALF PMNs were not evident until $12 \mathrm{~h}$ after ALI (Figure 2i-k).

\section{AT-RvD1 decreases lung edema and neutrophil accumulation after $A$ LI}

ALI from $\mathrm{HCl}$ instillation led to increased alveolar edema and inflammation (PMNs identified as $\mathrm{Ly}-6 \mathrm{G}^{+}$by immunostaining) (Figure 3a). Administration of AT-RvD1 (100 ng) either before (Figure 3b) or $2 \mathrm{~h}$ after (Figure 3c) intratracheal acid instillation decreased both alveolar edema and the numbers of $\mathrm{Ly}-6 \mathrm{G}^{+}$cells relative to control mice receiving only vehicle.

\section{Regulation of lung neutrophil recruitment by AT-RvD1}

To investigate the PMN recruitment pattern with AT-RvD1, its influence on intravascular and interstitial PMNs was determined (see Methods). Just before harvesting murine lungs $12 \mathrm{~h}$ after ALI, a PMN-specific Gr-1 antibody was injected intravenously to label intravascular PMNs. After BAL, the lungs were collected, prepared for FACS, and stained with a second PMN-specific antibody (NIMP-14R) (see Methods). PMNs were identified by forward/side scatter profiles and cells that were Gr- $1^{+}$NIMP$14 \mathrm{R}^{+}$were consistent with intravascular PMNs and cells that were Gr- $1^{-}$NIMP- $14 \mathrm{R}^{+}$were consistent with interstitial PMNs (Figure 4a). The percent interstitial and intravascular PMNs at this time point were decreased only slightly with AT-RvD1 (100 ng) relative to vehicle control, and these differences did not reach statistical significance (Figure $\mathbf{4 b}$ and $\mathbf{c}$ ). Of interest, the number of circulating PMNs $(1,255 \pm 108$ PMNs per $\mu l$, mean \pm s.e.m.) and monocytes (monos) (468 \pm 37 monos per $\mu$, mean \pm s.e.m.) were markedly increased $12 \mathrm{~h}$ after ALI relative to control animals without injury (243 \pm 56 PMNs per $\mu \mathrm{l}$; $153 \pm 57$ monos per $\mu \mathrm{l}, n=5, P<0.05$ ) (Figure $4 \mathbf{d}-\mathbf{g}$ ). Intravenous ATRvD1 significantly decreased the numbers of PMNs (866 \pm 59 PMNs per $\mu \mathrm{l}$, mean \pm s.e.m. $)$ and monos $(240 \pm 63$ monos per $\mu \mathrm{l}$, mean \pm s.e.m.) in the peripheral blood of acid-injured mice $(n=5$, $P<0.05$ ) (Figure 4d-g). 

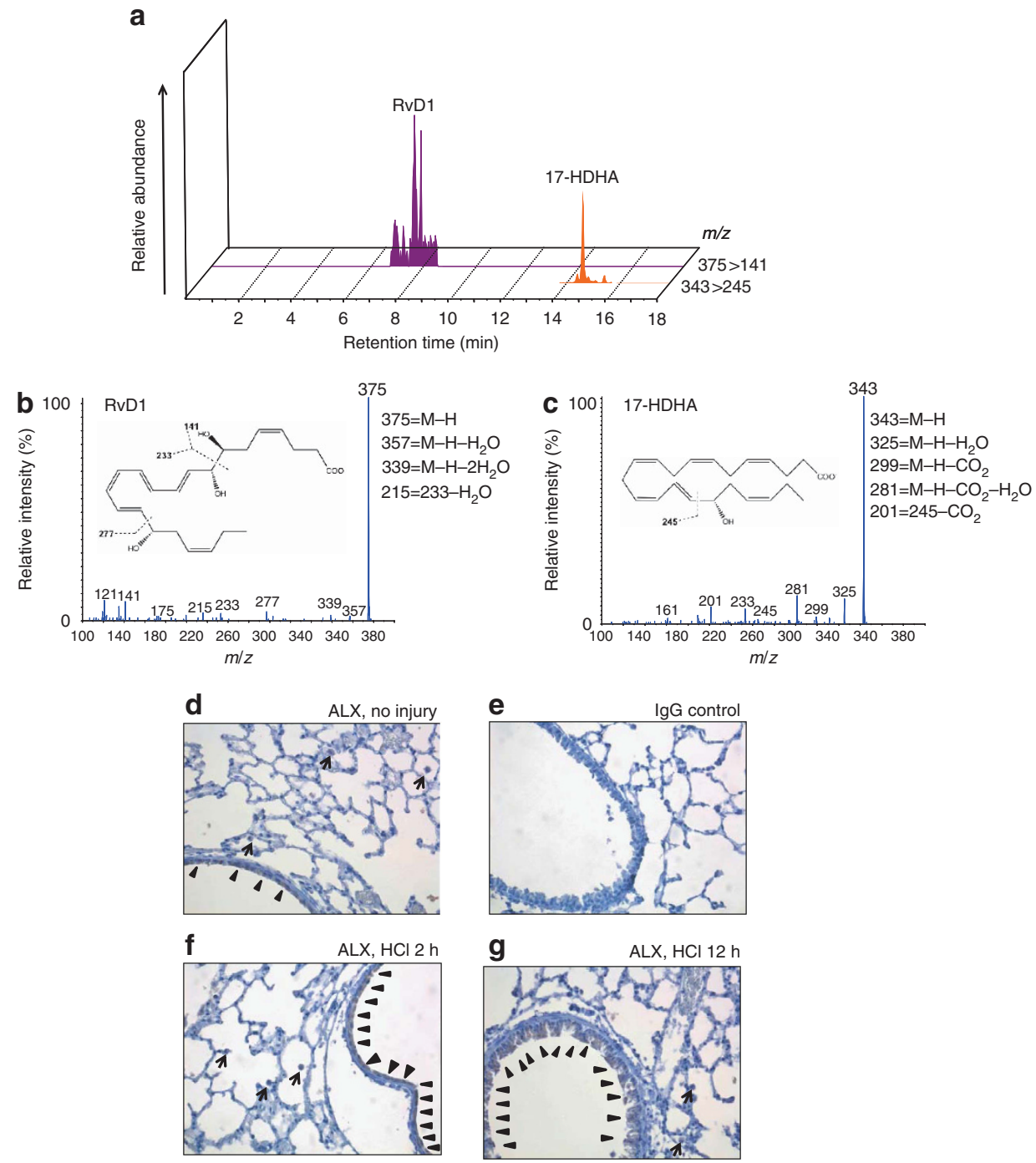

Figure 1 Resolvin D1 (RvD1), 17(S)-hydroxy-docosahexaenoic acid (17-HDHA), and ALX/FPR2 receptors are present in murine lungs after acute lung injury (ALI). Murine lung homogenates were obtained $12 \mathrm{~h}$ after $\mathrm{HCl}$-initiated ALI. Lipids were extracted and analyzed by liquid chromatography with tandem mass spectrometry (LC-MS/MS). (a) Multiple reaction monitoring (MRM) chromatograms (375>141 and 343>245) and representative tandem mass spectra of (b) RvD1 and (c) 17(S)-HDHA. Insets show structures and diagnostic fragmentation ions for each. Results are representative of $n=3$. Immunohistochemical analysis for ALX/FPR2 abundance was performed with lungs obtained (d) without injury exposed to ALX/FPR2 antibody $(1 / 50)$ or (e) IgG control antibody, and (f) $2 \mathrm{~h}$ and (g) $12 \mathrm{~h}$ after ALI. Arrowheads, epithelial cells; arrows, macrophages. Results are representative from $n=3$. Original magnification, $\times 400$.

\section{AT-RvD1 reduces lung resistance in ALI}

To investigate whether treatment with AT-RvD1 had a measurable effect on lung mechanics, we determined lung function in mechanically ventilated, anesthetized mice. Because of the unilateral and mild nature of the ALI in this model, marked changes in tissue elastance are not observed, ${ }^{8}$ and no significant differences were evident with AT-RvD1 relative to vehicle controls (Figure 5a). Of interest, the increased lung resistance $12 \mathrm{~h}$ after ALI in this model was significantly reduced by AT-RvD1 (100 ng) given either before $\left(0.65 \pm 0.05 \mathrm{cmH}_{2} \mathrm{O}^{*} \mathrm{sml}^{-1}\right.$, mean \pm s.e.m., $\left.n=12\right)$ or after ALI $\left(0.56 \pm 0.02 \mathrm{cmH}_{2} \mathrm{O}^{*} \mathrm{~s} \mathrm{ml}^{-1}\right.$, mean \pm s.e.m., $\left.n=6\right)$ in comparison to vehicle $\left(0.90 \pm 0.08 \mathrm{cmH}_{2} \mathrm{O}^{\star} \mathrm{s} \mathrm{ml}^{-1}\right.$, mean \pm s.e.m., $\left.n=10, P<0.05\right)$ (Figure 5b).

\section{AT-RvD1 enhances restitution of barrier function after ALI}

Because leakage permeability changes are integral to acute inflammation and ALI, and lung histology revealed that AT-RvD1 decreased alveolar edema (Figure 3), the actions of AT-RvD1 on both epithelial and endothelial barrier integrity were next determined. To measure the effects of AT-RvD1 on the restitution of epithelial barrier function after direct mucosal injury by $\mathrm{HCl}$, fluorescently labeled dextran was instilled intratracheally and $10 \mathrm{~min}$ later BALF and right ventricular blood were obtained for measurement of fluorescence (see Methods). The ratio of fluorescence in the serum to BALF provided an index of epithelial permeability. At $12 \mathrm{~h}$ after acid instillation, epithelial permeability is increased and control mice receiving only vehicle gave serum/BALF fluorescence ratio of $0.36 \pm 0.07$ 

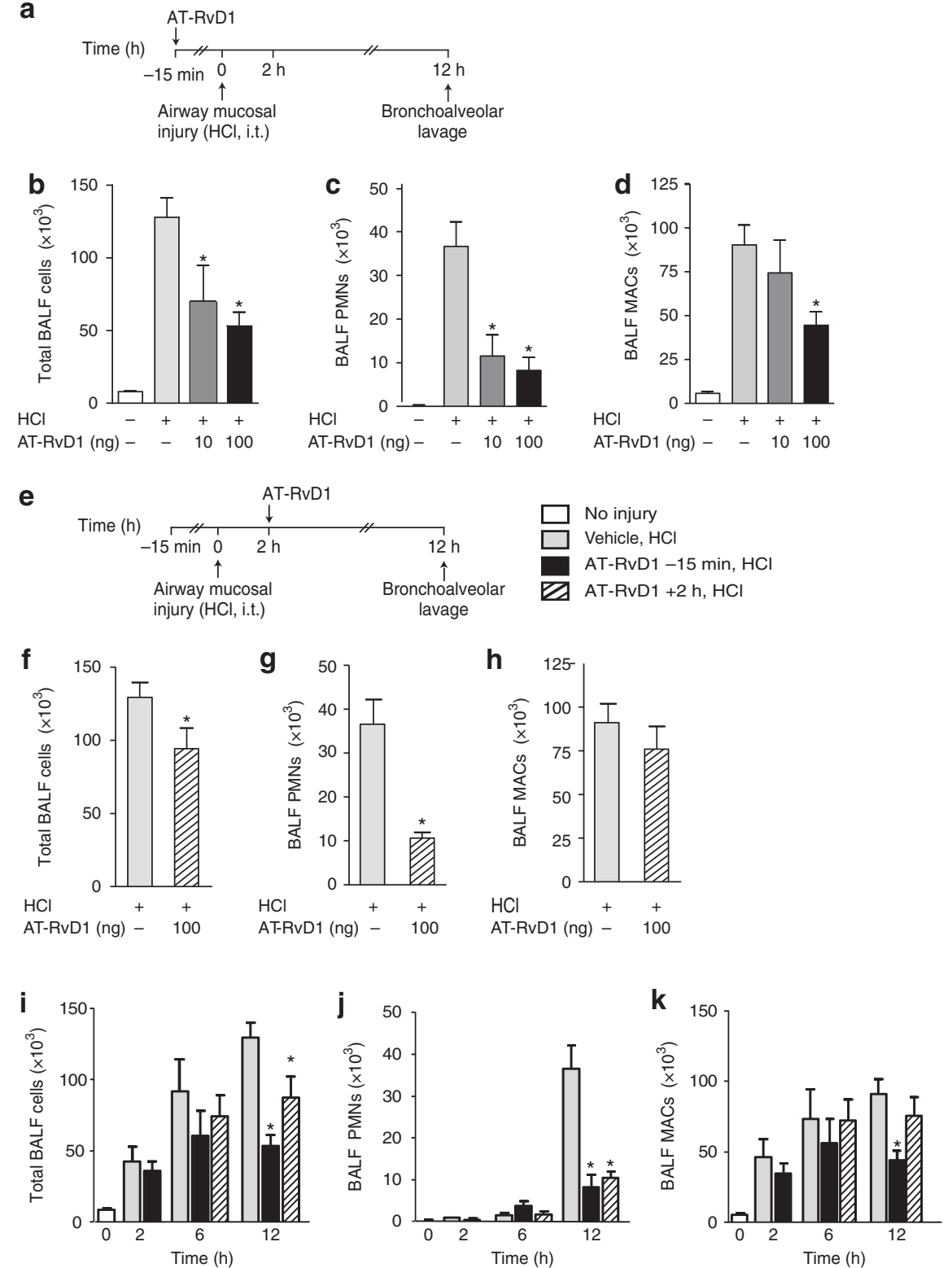

Figure 2 Aspirin-triggered resolvin D1 (AT-RvD1) reduces leukocyte recruitment after airway mucosal injury. (a) AT-RvD1 (10, 100 ng) or vehicle alone $(0.1 \%$ ethanol in $0.9 \% \mathrm{NaCl}(\mathrm{vol} / \mathrm{vol}))$ were given intravenously $15 \mathrm{~min}$ before acute lung injury (ALI). After $12 \mathrm{~h}$, bronchoalveolar lavage (BAL) was performed and (b) total cells, (c) polymorphonuclear leukocytes (PMNs), and (d) macrophages (MACs) in BAL fluids (BALFs) were enumerated (see Methods). (e) In a separate cohort, the impact of AT-RvD1 (100 ng) or vehicle $(0.1 \%$ ethanol in $0.9 \% \mathrm{NaCl}$ (vol/vol)) given $2 \mathrm{~h}$ after ALI was determined on BALF (f) total cells, (g) PMNs, and (h) MACs $12 \mathrm{~h}$ after ALI was initiated. (i-k) Early leukocyte recruitment to the lung was assessed in the presence of AT-RvD1 (100 ng, intravenously) or vehicle $(0.1 \%$ ethanol in $0.9 \% \mathrm{NaCl}(\mathrm{vol} / \mathrm{vol}))$ given $15 \mathrm{~min}$ before or $2 \mathrm{~h}$ after $\mathrm{ALI}$. Values represent the mean \pm s.e.m. for $n \geqslant 5$ mice. ${ }^{*} P<0.05$ vs. vehicle group. I.t., intratracheally.

(mean \pm s.e.m., $n=5$ ) (Figure 5c). AT-RvD1 (100 ng) significantly improved epithelial barrier disruption $12 \mathrm{~h}$ after mucosal injury with decreased serum/BALF fluorescence ratios relative to vehicle controls when the compound was given either before injury $(0.08 \pm 0.03$, mean \pm s.e.m., $n=3, P<0.05)$ or $2 \mathrm{~h}$ after injury $(0.09 \pm 0.02$, mean \pm s.e.m., $n=5, P<0.01)$ (Figure 5c). Direct actions of the compound on epithelial barrier integrity in vitro were not identified, as AT-RvD1 (1-100 nM) did not significantly change the trans-epithelial electrical resistance of Calu-3 human bronchial epithelial cells in culture. AT-RvD1 improved vascular permeability changes in vivo, as the amount of Evans blue dye that extravasated into BALF was significantly decreased in mice receiving AT-RvD1 $(100 \mathrm{ng})$ either before injury $\left(4.7 \pm 0.2 \mu \mathrm{g} \mathrm{ml}^{-1}\right.$, mean \pm s.e.m., $n=3, P<0.01)$ or $2 \mathrm{~h}$ after injury $\left(4.6 \pm 0.8 \mu \mathrm{g} \mathrm{ml}^{-1}\right.$, mean \pm s.e.m., $n=3, P<0.05)$ in comparison to vehicle control mice $\left(8.5 \pm 0.8 \mu \mathrm{g} \mathrm{ml}^{-1}, n=4\right)$ (Figure 5d).

\section{AT-RvD1 decreases neutrophil-platelet interactions}

Vascular inflammation with heterotypic interactions between PMNs, platelets, and endothelial cells are important early 


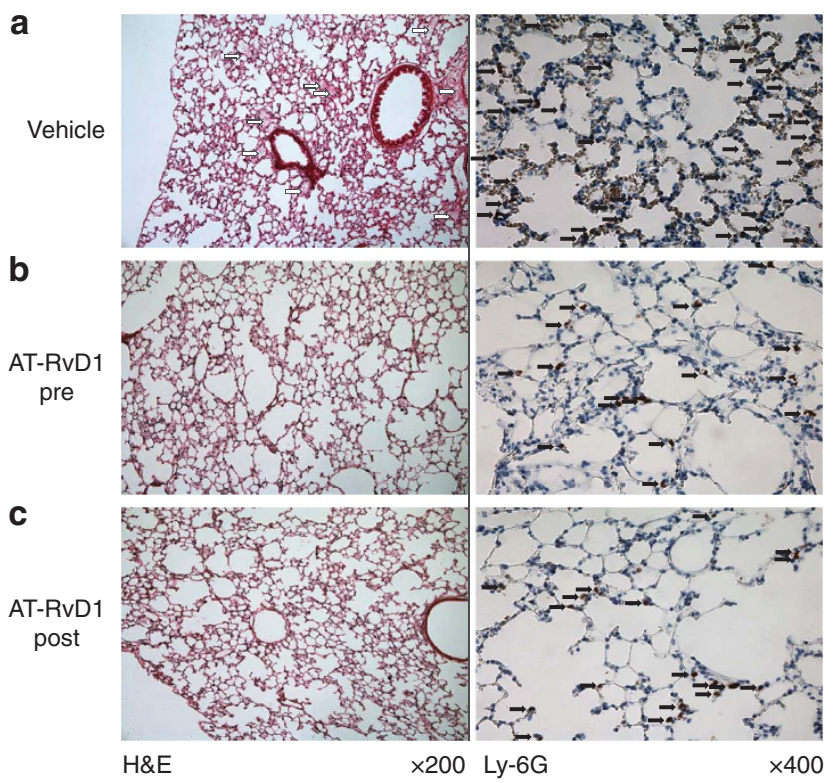

Figure 3 Aspirin-triggered resolvin D1 (AT-RvD1) decreased the lung histopathological changes after acid-initiated acute lung injury (ALI). Lungs were obtained $12 \mathrm{~h}$ after ALI from mice that were exposed to vehicle $(0.1 \%$ ethanol in $0.9 \% \mathrm{NaCl}$ (vol/vol), upper row (a)), AT-RvD1 (100ng) before ALI (middle row (b)) or AT-RvD1 (100 ng) $2 \mathrm{~h}$ after acid injury (lower row (c)). Lung tissue sections were prepared from fixed, paraffin-embedded organs and stained with either hematoxylin and eosin (H\&E) (left) or Ly-6G (1/100 dilution) (right) (see Methods). Alveolar edema (white arrows) and leukocytes (black arrows) are highlighted in representative images $(n=3)$. Original magnification of $\mathrm{H} \& \mathrm{E}$ was $\times 200$ and $L y-6 \mathrm{G}$ was $\times 400$.

events in ALI. ${ }^{19,20}$ To determine if AT-RvD1 regulated these cell-cell interactions, blood was obtained from mice given either AT-RvD1 (100 ng) or vehicle alone and analyzed by flow cytometry (Figure 5e). PMNs were identified by their physical properties and Ly-6G staining. PMN-platelet interactions were identified as PMNs $\left(\mathrm{Ly}-6 \mathrm{G}^{+}\right.$) that were also $\mathrm{CD} 41^{+}$(platelets), and AT-RvD1 decreased the percent $\mathrm{Ly}-6 \mathrm{G}^{+} \mathrm{CD} 41^{+}$cells. P-selectin (CD62P) plays important roles in PMN-platelet interactions in ALI, ${ }^{19,20}$ and AT-RvD1 decreased the numbers of Ly- $6 \mathrm{G}^{+} \mathrm{CD}^{2} 2 \mathrm{P}^{+}$cells (Figure 5e). Western blot analysis of lung tissue homogenates also revealed decreased expression of both CD62P and its granulocyte receptor CD24 in mice receiving AT-RvD1 (Figure 5f).

\section{Endogenous airway epinephrine levels are enhanced by AT-RvD1}

Because AT-RvD1 decreased lung resistance after ALI (Figure 5b), BALF epinephrine levels were next determined using a specific and sensitive enzyme-linked immunosorbent assay (see Methods). BALF epinephrine levels were significantly increased in mice that had received AT-RvD1 (100 ng) either before injury (201.4 $\pm 13.2 \mathrm{pg} \mathrm{ml}^{-1}$, mean \pm s.e.m.) or after injury $\left(310 \pm 37.1 \mathrm{pg} \mathrm{ml}^{-1}\right.$, mean \pm s.e.m. $)$ in comparison to vehicle ( $146.7 \pm 8.4 \mathrm{pg} \mathrm{ml}^{-1}$, mean \pm s.e.m., $\left.n \geqslant 6, P<0.01\right)$ (Figure 6a).

\section{AT-RvD1 decreases pro-inflammatory mediator release after ALI}

To determine the impact of AT-RvD1 on inflammatory mediators after ALI, BALF levels of select cytokines, chemokines, and lipid mediators were determined. BALF tumor growth factor- $\beta$ was significantly increased when AT-RvD1 was administered $2 \mathrm{~h}$ after ALI (Figure $\mathbf{6 b}$ ). BALF levels of the counter-regulatory mediators lipoxin $\mathrm{A}_{4}\left(\mathrm{LXA}_{4}\right)$ and interleukin (IL)-10 did not increase with AT-RvD1 relative to vehicle; however, the $\mathrm{LXA}_{4}$ and IL-10 levels were significantly different between the two ATRvD1 treatment strategies (Figure $\mathbf{6 c}$ and $\mathbf{d}$ ). When AT-RvD1 was given before $\mathrm{HCl}, \mathrm{LXA}_{4}$ and IL-10 levels decreased, but there was no significant change in $\mathrm{LXA}_{4}$ and IL-10 levels when AT-RvD1 was given $2 \mathrm{~h}$ after injury (Figure $6 \mathrm{c}$ and $\mathbf{d}$ ). Several pro-inflammatory mediators were significantly decreased in AT-RvD1-treated mice, including IL- $1 \beta$, IL-6, keratinocyte chemoattractant, and tumor necrosis factor- $\alpha$ (Figure $\mathbf{6 e - h}$ ). No significant changes were observed in BALF chemokine (C-C motif) ligand 3 , chemokine ( $\mathrm{C}-\mathrm{X}-\mathrm{C}$ motif) ligand 2 , chemokine (C-X-C motif) ligand 10 , and leukotriene $\mathrm{B}_{4}$ (Figure 6i-1). In addition, levels of 8 -isoprostane, a sensitive marker of oxidative stress, are low at this time point in this model, ${ }^{8}$ and there were no significant changes apparent with AT-RvD1 (data not shown).

\section{AT-RvD1 decreases NF- $\mathrm{KB}$ p65 translocation after ALI}

Because AT-RvD1 markedly decreased several pro-inflammatory cytokines, the impact of AT-RvD1 on NF- $\kappa \mathrm{B}$ activation and the cellular targets for AT-RvD1 counter-regulatory actions were next determined by immunohistochemistry of lung sections. NF- $\kappa \mathrm{B}$ activation and nuclear translocation was identified using a phosphorylated p65 (serine 276) antibody (see Methods). There was an increase in NF- $\kappa B$ phosphoserine p65 abundance and nuclear translocation in airway epithelial cells and lung macrophages that was evident $2 \mathrm{~h}$ after ALI (Figure $7 \mathbf{a}-\mathbf{c}$ ) and markedly increased $12 \mathrm{~h}$ after ALI (Figure 7d). Both dosing strategies for AT-RvD1 decreased the abundance of NF- $\kappa B$ phosphoserine p65 in epithelial cells and macrophages $12 \mathrm{~h}$ after $\mathrm{HCl}$ (Figure $7 \mathrm{e}$ and $\mathbf{f}$ ).

\section{DISCUSSION}

Alveolar edema and PMN recruitment and activation are early events in acute mucosal inflammation and ALI/ARDS. ${ }^{1}$ These cellular events are subject to regulation by lipid mediators, ${ }^{6,19}$ and here $\mathrm{RvD} 1$ was generated after $\mathrm{HCl}$-initiated ALI. The RvD1 receptor ALX/FPR2 is expressed on PMNs, monos, macrophages, and airway epithelial cells. ${ }^{17,21-23}$ Murine ALX/FPR2 lung expression was evident in airway epithelial cells and macrophages, and its abundance markedly increased after ALI. The RvD1 epimer AT-RvD1 displayed properties of both an antiinflammatory and pro-resolving mediator in decreasing the severity of $\mathrm{HCl}$ acid-initiated ALI. Given either before or shortly after mucosal injury, AT-RvD1 reduced several parameters of alveolar edema $12 \mathrm{~h}$ after ALI, including by histological evidence, and measures of epithelial and endothelial permeability. There was an increase in lung tissue resistance after intratracheal administration of $\mathrm{HCl}$ that was reduced by AT-RvD1 and 
a
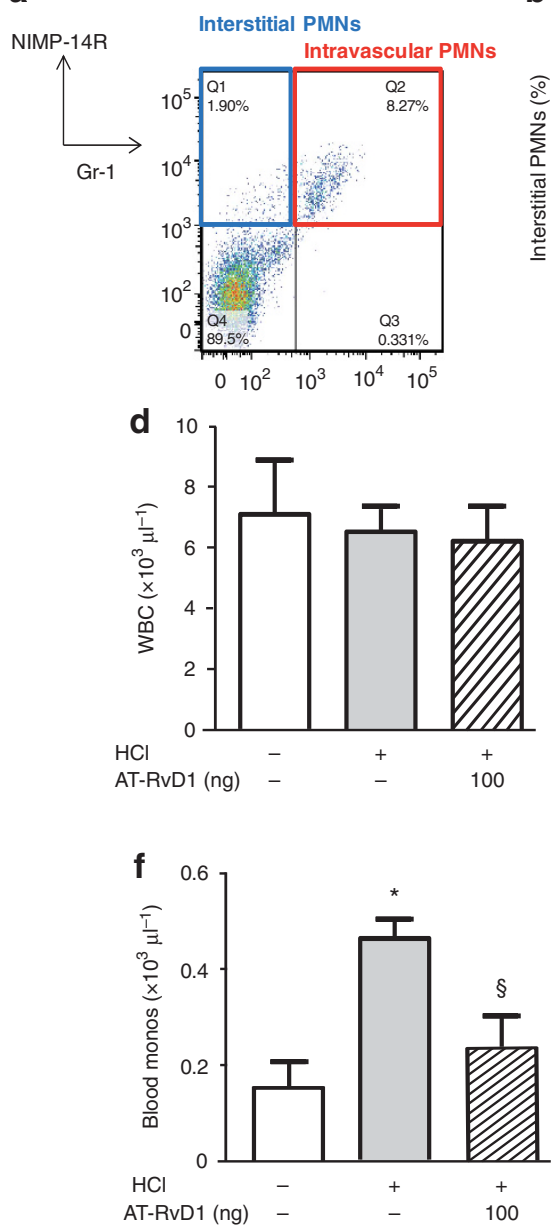

b

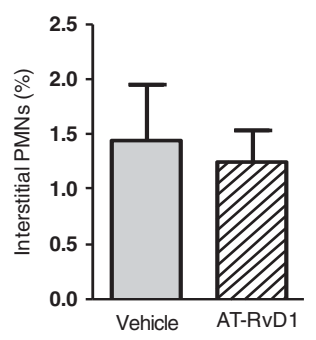

C

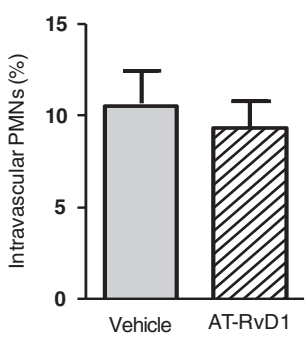

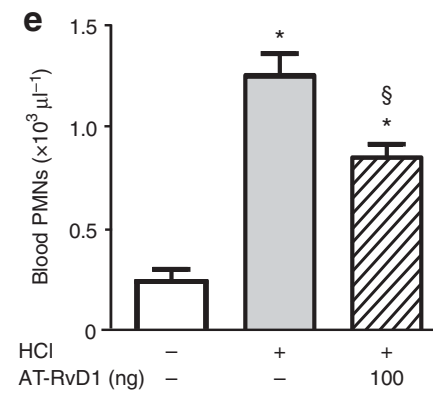

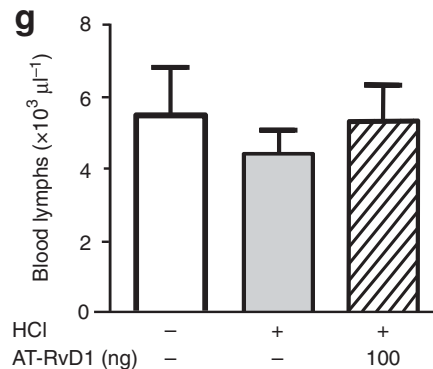

Figure 4 Aspirin-triggered resolvin D1 (AT-RvD1) decreased peripheral blood leukocytosis to decrease lung recruitment after acute lung injury (ALI). (a) Intravascular polymorphonuclear leukocytes (PMNs) (NIMP-14R+ ${ }^{+} \mathrm{Gr}-1^{+}$) were distinguished from interstitial PMNs (NIMP-14R+ Gr-1-) by FACS in (b and $\mathbf{c}$ ) lungs obtained $12 \mathrm{~h}$ after ALI from mice that were exposed to vehicle or AT-RvD1 (100 ng) $2 \mathrm{~h}$ after intratracheal acid instillation. Whole blood was collected $12 \mathrm{~h}$ after ALI and (d) total leukocytes (WBC), (e) PMNs, (g) monocytes (monos), and (h) lymphocytes (lymphs) were enumerated. Values represent the mean \pm s.e.m. for $n \geqslant 3$ mice. ${ }^{\star} P<0.05$ vs. control group. $\S P<0.05$ vs. vehicle group.

associated with AT-RvD1-mediated increases in endogenous BALF epinephrine levels. In addition to these actions on mucosal tissue-resident cells, AT-RvD1 also displayed potent leukocytedirected actions, including decreased circulating PMNs and monos, vascular PMN-platelet heterotypic interactions, airway PMN infiltration, BALF pro-inflammatory mediator levels, and NF- $\kappa B$ p 65 activation in airway epithelial cells and macrophages. Taken together, these findings indicate that in lung tissue and inflammatory cells AT-RvD1 harnessed several cell type-specific protective mechanisms to decrease ALI severity. AT-RvDl's regulation of vascular PMN-platelet interactions, permeability changes, and epinephrine levels emphasize the importance of vascular inflammatory events in the pathogenesis of early ALI.

Retrospective analyses of patients with ARDS have identified aspirin as a protective exposure that decreases ARDS morbidity and mortality. ${ }^{16}$ In addition to inhibiting platelet function, aspirin triggers the formation of specific pro-resolving mediators, ${ }^{24}$ including AT-RvD1 from DHA in inflamed murine and human tissues. ${ }^{14}$ In murine models of acute inflammation, AT-RvD1 decreases the production of pro-inflammatory mediators and regulates leukocyte trafficking to inflammatory sites (reviewed in ref. 25), including in experimental models of arthritis and colitis. ${ }^{26,27}$ Here, AT-RvD1 decreased lung PMN infiltration and decreased inflammatory responses by tissue-resident cells, including endothelial and epithelial barrier function and levels of several pro-inflammatory mediators in the injured lung. AT-RvD1 2 hours after injury gave similar protection as a dose given $15 \mathrm{~min}$ before injury, indicating that AT-RvD1 could accelerate the resolution of ALI.

Neutrophil activation can cause bystander tissue damage that contributes to the pathogenesis of ALI/ARDS; ${ }^{28}$ thus, decreasing lung PMN accumulation and pro-inflammatory mediators would be predicted to have synergistic benefit for tissue protection and catabasis after acid injury. To this end, inhibition of PMN function in animal studies attenuates lung injury induced by models of gastric acid aspiration. ${ }^{29,30} \mathrm{RvD} 1$ transduces potent anti-inflammatory actions for PMNs. Single-cell PMN assays in microfluidic chambers have uncovered direct PMN actions 
a

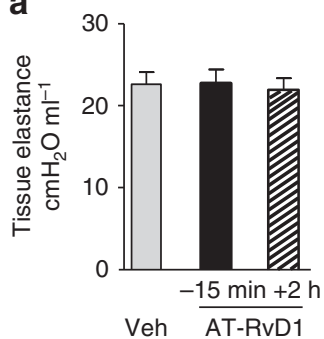

b

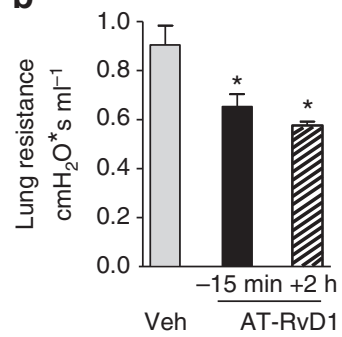

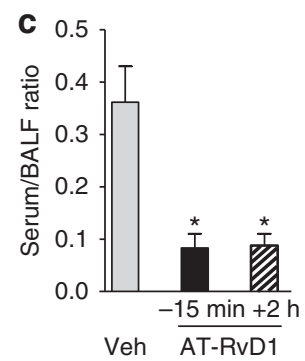

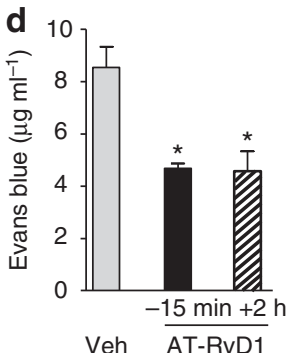

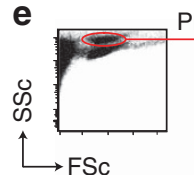
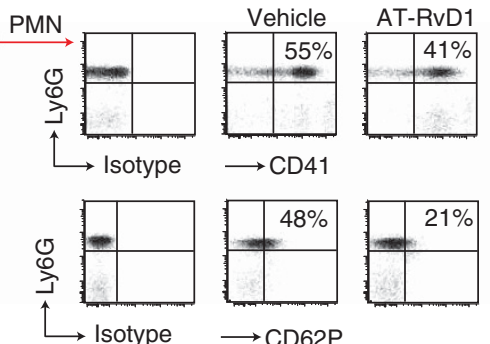
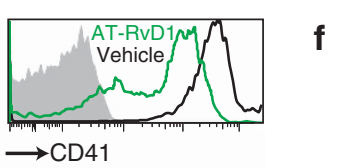

f

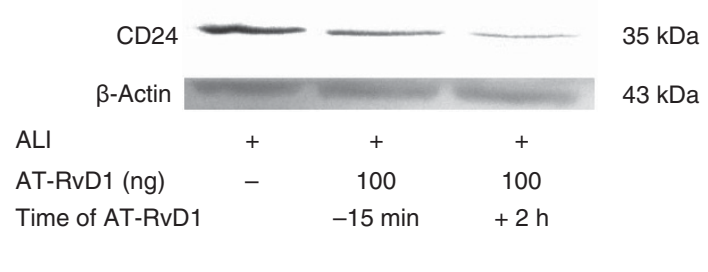

Figure 5 Counter-regulatory actions for aspirin-triggered resolvin D1 (AT-RvD1) on lung mechanics, barrier integrity, and vascular inflammation after acute lung injury (ALI). At $12 \mathrm{~h}$ after ALI in mice exposed to AT-RvD1 (100 ng) or vehicle, lung mechanics were determined using a flexiVent mouse ventilator, including (a) tissue elastance and (b) lung resistance (see Methods). Values represent the mean \pm s.e.m. for $n \geqslant 6$ mice. ${ }^{*} P<0.05$ vs. vehicle group. (c) Epithelial and (d) endothelial barrier integrity $12 \mathrm{~h}$ after ALI was determined in separate animals with intratracheal fluorescein isothiocyanate (FITC)-dextran and intravenous Evans blue dye, respectively (see Methods). Values represent the mean \pm s.e.m. for $n \geqslant 3$ mice. ${ }^{*} P<0.05$ vs. vehicle group. (e) Cell-cell interactions between polymorphonuclear leukocytes (PMNs) and platelets were monitored by flow cytometry. Representative ( $n=3$ ) detection of $\mathrm{Ly} 6 \mathrm{G}{ }^{+} \mathrm{CD} 41^{+}$and $\mathrm{Ly} 6 \mathrm{G}{ }^{+} \mathrm{CD} 62 \mathrm{P}^{+}$cells in murine blood $12 \mathrm{~h}$ after ALI. The percentages of positive cells are indicated at the top of the respective gates. (f) Representative $(n \geqslant 3)$ western analysis for abundance in lung homogenates $12 \mathrm{~h}$ after ALI of CD62P and CD24 (see Methods). FSc, forward scatter; Ssc, side scatter.

for RvD1, but not the parent fatty acid DHA, to stop rapidly PMN migration towards the chemoattractant IL- $8,{ }^{31}$ and RvD1 can regulate PMN lung recruitment in response to lipopolysaccharide. ${ }^{32}$ Here, early administration of AT-RvD1 appeared to reduce the amplitude and duration of acid-initiated ALI and together with AT-RvD1's regulation of NF- $\kappa \mathrm{B}$ activation suggests that the compound's reduction in airway inflammation is secondary to both direct actions on leukocytes and indirect actions to decrease the levels of inflammatory cytokines. In addition, AT-RvDl's actions on leukocytes to decrease their lung recruitment in conjunction with regulation of vasoactive mediators likely accounts for the decrease in alveolar edema with AT-RvD1. In this regard, at ocular mucosal surfaces, RvD1 decreases cysteinyl leukotrienes, ${ }^{33}$ which also play a pivotal role in vascular permeability in $\mathrm{HCl}$-induced ALI. ${ }^{8}$

LXs are arachidonic acid-derived mediators that display antiinflammatory and pro-resolving actions, including inhibition of in vivo PMN activation, cytokine release, and angiogenesis (reviewed in ref. 25). These protective actions are mediated, in part, by the $\mathrm{LXA}_{4}$ receptor ALX/FPR2 that can also serve as an anti-inflammatory receptor for RvD1 and AT-RvD1. ${ }^{17,18,23}$ ALX/FPR2 receptor expression is increased in vitro after airway epithelial injury, ${ }^{21}$ and transgenic expression of human ALX/ FPR2 receptors is protective from $\mathrm{HCl}$-initiated ALI. ${ }^{15}$ Here, ALX/FPR2 receptor expression increased in vivo $12 \mathrm{~h}$ after ALI in both airway epithelial cells and lung macrophages, and ATRvD1 administration markedly decreased NF- $\kappa \mathrm{B}$ activation in these ALX/FPR2-expressing cells. Although apoptotic PMNs are difficult to identify in this model of ALI, RvD1 in other model systems are potent stimuli for macrophage efferocytosis during tissue catabasis ${ }^{17}$ and can promote host catabatic responses that in conjunction with antibiotics promotes the resolution of bacterial infection. ${ }^{34}$ Of interest, BALF levels of $\mathrm{LXA}_{4}$ were significantly decreased by AT-RvD1 administration before injury, which were significantly lower than $\mathrm{LXA}_{4}$ levels in mice that received AT-RvD1 after injury. There are two implications of this finding. First, AT-RvD1's protective actions are independent of $\mathrm{LXA}_{4}$ generation, and second, the early events in host acute inflammatory responses to tissue injury are critical to endogenous biosynthesis of $\mathrm{LXA}_{4}$. Early pro-inflammatory mediator formation is pivotal to lipid mediator class switching for the later generation of anti-inflammatory mediators, such as $\mathrm{LXA}_{4} \cdot{ }^{15,35}$ Administration of AT-RvD1 before injury dampened the early pro-inflammatory mediator production with reductions in endogenous $\mathrm{LXA}_{4}$ generation $12 \mathrm{~h}$ later. Because AT-RvD1 resists rapid inactivation ${ }^{14}$ and promoted ALI resolution, the decrements in $\mathrm{LXA}_{4}$ generation did not have adverse consequences on lung catabasis. This is in sharp contrast with chemical inhibitors of early inflammatory mediator generation, such as cyclooxygenase-2-specific inhibitors or 5-lipoxygenase inhibition, which can be resolution "toxic" for tissue injury or inflammation. ${ }^{15,36}$

$\mathrm{P}$-selectin (CD62P) is expressed by activated endothelial cells and platelets as a molecular regulator of heterotypic PMNplatelet and platelet-endothelial cell interactions. ${ }^{37}$ During secondary capture, PMNs interact with circulating or endothelial 

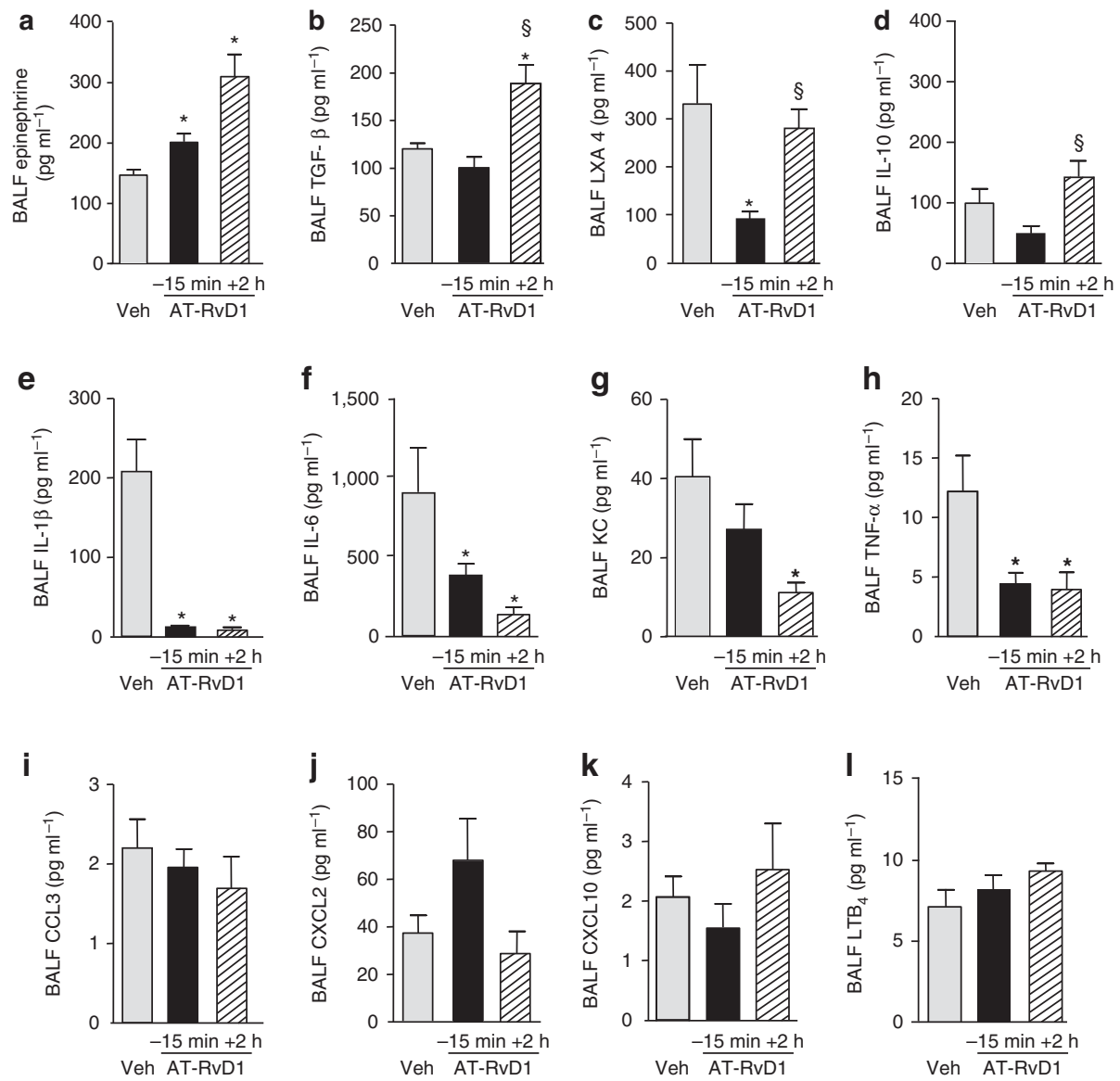

Figure 6 Impact of aspirin-triggered resolvin D1 (AT-RvD1) on inflammatory mediator levels after acute lung injury (ALI). Aliquots of bronchoalveolar lavage fluids (BALFs) obtained $12 \mathrm{~h}$ after ALI were analyzed by enzyme-linked immunosorbent assay (ELISA) (a, c, d, and I) or cytokine bead array (b, e-k). Values represent the mean \pm s.e.m. for $n \geqslant 3$ mice. ${ }^{*} P<0.05$ vs. vehicle (Veh) group. ${ }^{\S} P<0.05$ vs. $100 \mathrm{ng}$ AT-RvD1 group 15 min before ALI. CCL3, chemokines (C-C motif) ligand 3; CXCL2, chemokine (C-X-C motif) ligand 2; IL, interleukin; KC, keratinocyte chemoattractant; $\mathrm{LXA}_{4}$, lipoxin $\mathrm{A}_{4} ;$ LTB $_{4}$, leukotriene $\mathrm{B}_{4}$; TNF- $\alpha$, tumor necrosis factor- $\alpha$.

adherent platelets to augment $\mathrm{PMN}$-endothelial interactions in inflamed vascular beds for PMN tissue recruitment. ${ }^{38}$ Of note, platelet depletion leads to diminished leukocyte recruitment in tissue inflammation and ALI. ${ }^{19}$ As a measure of vascular inflammation, heterotypic cell-cell interactions between PMNs and platelets were monitored here in blood from the right ventricle, just before entering the pulmonary vasculature. The peripheral blood leukocytosis induced by ALI was decreased by AT-RvD1, and flow cytometry revealed a substantial reduction in PMNplatelet interactions with AT-RvD1. In addition, the expression of P-selectin (CD62P) and its PMN ligand CD24 was decreased by AT-RvD1 in lung homogenates after ALI. Blocking P-selectin-dependent platelet-PMN interactions is highly protective in a similar model of acid-initiated ALI. ${ }^{19}$ In addition to circulating PMNs and platelet-PMN interactions, resolvins can also decrease ADP-stimulated platelet aggregation. ${ }^{39}$ These findings suggest that AT-RvD1's mucosal protection in ALI resulted, in part, from its actions on PMNs that decreased PMN recruitment from bone marrow to lung and decreased PMN-mediated disruption of lung barrier integrity by regulating PMN interactions in the vasculature with platelets. Taken together, these findings indicate the presence of unique homeostatic pathways for DHA-derived bioactive mediators in the lung.

AT-RvD1 is a potent regulator of mucosal inflammation and can promote an array of protective responses for lung catabasis after ALI. In addition, it is notable that the pharmacologically active dose of AT-RvD1 administered intravenously was $<100 \mathrm{ng}$ per mouse, or $\sim 0.005 \mathrm{mg} \mathrm{kg}^{-1}$, providing evidence of this compound's potent anti-inflammatory and pro-resolving actions. Enteric feeding with DHA can markedly reduce ALI/ARDS morbidity and mortality, in part, via marked decreases in lung PMNs and inflammatory mediators. ${ }^{9,10}$ The potential for lung protection by DHA-derived mediators is also supported by evidence from fat- 1 transgenic mice that are protected from $\mathrm{ALI}^{40}$ and allergic airway inflammation. ${ }^{41}$ These transgenic mice express the Caenorhabditis elegans $\omega-3$ desaturase fat-1 gene and can endogenously generate $\omega-3$ polyunsaturated fatty acids from $\omega-6$ polyunsaturated fatty acids ${ }^{39}$ and resolvins. ${ }^{41}$ Moreover, AT-RvD1's epimer RvD1 displays protective actions in a lipopolysaccharide model of acute lung inflammation and injury. ${ }^{32}$

In summary, our findings have uncovered new roles for AT$\mathrm{RvD1}$ in promoting resolution of acid-initiated experimental 

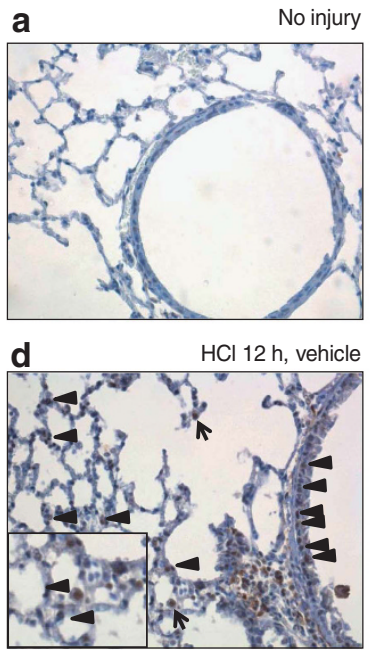

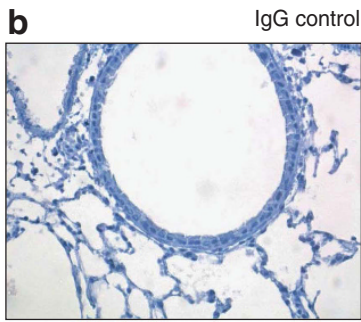

$\mathrm{HCl} 12$ h, AT-RvD1 (pre)

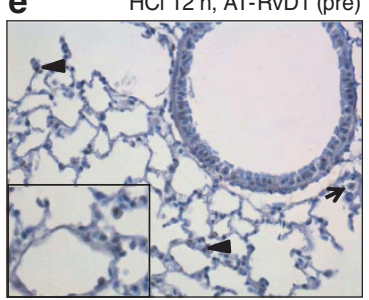

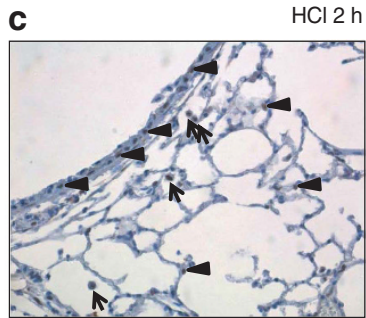

$\mathrm{HCl} 12 \mathrm{~h}, \mathrm{AT}-\mathrm{RvD} 1$ (post)

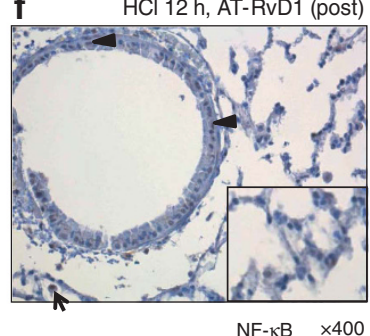

Figure 7 Aspirin-triggered resolvin D1 (AT-RvD1) decreased acute lung injury (ALI)-induced nuclear factor- $\mathrm{B}$ (NF- $\mathrm{kB})$ p65 activation and nuclear translocation in murine lung. Immunohistochemical analysis to detect NF- $\mathrm{BB}$ localization was performed by staining lung tissue (a) without injury using NF-kB-phosphorylated p65 antibody (phospho-S276, 1/50) or (b) IgG control antibody and (c) $2 \mathrm{~h}$ after ALI. Lungs were also obtained $12 \mathrm{~h}$ after ALI from mice exposed to (d) vehicle or (e, f) AT-RvD1 (100 ng). Insets, higher magnification to show nuclear staining. Arrowheads, epithelial cells; arrows, macrophages. Results are representative from $n=3$. Original magnification $\times 400$.

ALI. In this study, the DHA-derived lipid mediator AT-RvD1 decreased acid-initiated ALI by direct actions on circulating leukocytes and platelets with lung protective effects on barrier function, PMN accumulation, and pro-inflammatory mediator generation. Expression of ALX/FPR2 receptors and AT-RvD1's regulation of NF- $\kappa \mathrm{B}$ in lung mucosal epithelial cells and macrophages suggest important roles for these cells in lung tissue catabasis that will serve as a focus of future studies. Taken together, these results suggest a potential new therapeutic approach to the important clinical problem of ALI/ARDS that emphasizes natural resolution signaling pathways.

\section{METHODS}

Acid-initiated ALI and treatment with AT-RVD1. All mice were maintained under specific pathogen-free conditions. All studies were reviewed and approved by the Harvard Medical Area standing committee on animals (protocol no. 03618). As in Fukunaga et al. ${ }^{15}$ hydrochloric acid $(0.1 \mathrm{~N} \mathrm{HCl}, \mathrm{pH} 1.5,50 \mu \mathrm{l}$, endotoxin free; Sigma-Aldrich, St Louis, MO) was instilled selectively into the left mainstem bronchus of anesthetized mice (FVB, male, 10-12 weeks; Charles River, Wilmington, MA) via a $24-\mathrm{G}$ angiocatheter inserted intratracheally. Some mice received AT-RvD1 (10 or $100 \mathrm{ng}$ ) or vehicle $(0.1 \%(\mathrm{vol} / \mathrm{vol})$ ethanol) in saline by intravenous injection either 15 min before ALI or $2 \mathrm{~h}$ after ALI was initiated. At select time points $(2,6$, and $12 \mathrm{~h})$ after acid instillation, bilateral BAL was performed with two aliquots of $1 \mathrm{ml}$ of phosphate-buffered saline plus $0.6 \mathrm{~mm}$ ethylenediaminetetraacetic acid.

Leukocyte recruitment after ALI. The total cell counts and leukocyte differential in BALFs were determined as described previously. ${ }^{8}$ Briefly, total cells in BALFs were counted using a hemocytometer, and a leukocyte differential count was performed after Wright-Giemsa staining.

To determine intravascular and interstitial PMNs, FITC-Gr-1 (Clone RB6-8C5; eBioscience, San Diego, CA) was intravenously injected 5 min before lung harvest (similar to that in ref. 19). After BAL, the lungs were removed, dispersed, and passed through a $70-\mu \mathrm{m}$ cell strainer (as in ref. 42). PMNs were identified during flow cytometry by their forward/ side scatter profiles and their expression of NIMP-14R (Santa Cruz Biotechnology, Santa Cruz, CA) and Gr-1. Isotype control antibodies were used for compensation for nonspecific binding. The FITC-Gr-1 Ab was used to identify PMNs in the intravascular (NIMP- $14 \mathrm{R}^{+} \mathrm{Gr}-1^{+}$) and interstitial (NIMP-14R $\mathrm{R}^{+} \mathrm{Gr}^{-}$) compartments. Flow cytometry was performed using a BD FACS Canto II flow cytometer (Becton Dickinson, San Jose, CA) and data were analyzed using the FlowJo software (Tree Star, Ashland, OR).

Complete blood count and differential count. Murine blood was drawn from the inferior vena cava and lysed with RBC lysis buffer (eBioscience). Leukocytes were counted with a hemocytometer and differential count was performed after Wright-Giemsa staining. For the leukocyte differential, a minimum of 200 cells were counted per slide.

Neutrophil-platelet interactions. Whole blood was collected from mice that received either AT-RvD1 (100 ng) or vehicle $2 \mathrm{~h}$ after ALI. Blood was lysed using 10 volumes of red blood cell lysis buffer (eBioscience) as per the manufacturer's instructions. For flow cytometry, cells were blocked with CD16/32 Fc block (eBioscience) and then stained with Ly6G (eBioscience), CD41 (eBioscience), or CD62P (eBioscience) for 20 min on ice. CD24 and P-selectin protein abundance in lung homogenates was determined by western blot analyses. $\beta$-Actin was used as a control. Antibodies were used as per the manufacturer's recommended concentrations $\left(1 \mu \mathrm{g} \mathrm{ml}^{-1}\right)$ (Santa Cruz Biotechnology).

Identification of RvD1 in murine lung. FVB mouse lungs were suspended in $1.0 \mathrm{ml}$ cold methanol and gently ground, followed by protein precipitation for $12 \mathrm{~h}$. Samples were next extracted by solid-phase extraction column and methyl formate fractions were taken for liquid chromatography with tandem mass spectrometry-based lipidomics. Liquid chromatography with tandem mass spectrometry was performed with an Agilent 1100 HPLC (Agilent Technologies, Santa Clara, CA) equipped with an Agilent Eclipse Plus C-18 column $(4.6 \mathrm{~mm} \times 50 \mathrm{~mm} \times 1.8 \mu \mathrm{m})$ paired with an ABI Sciex Instruments 5500 QRAP linear ion trap triple quadrupole mass spectrometer (Applied Biosystems, Foster City, $\mathrm{CA})$. Instrument control and data acquisition were performed using 
the AnalystTM 1.5 software (Applied Biosystems). The mobile phase consisted of methanol/water/acetic acid (55/45/0.01; vol/vol/vol) and was ramped to $88 / 12 / 0.01$ ( $\mathrm{vol} / \mathrm{vol} / \mathrm{vol}$ ) after $10 \mathrm{~min}, 100 / 0 / 0.01$ ( vol/vol/vol) after $18 \mathrm{~min}$, and 55:45:0.01 (vol/vol/vol) after $1 \mathrm{~min}$ to wash and equilibrate the column. Mass spectrometry analyses were carried out in negative ion mode using multiple reaction monitoring of established specific transitions for 17(S)-hydroxy-DHA $(\mathrm{m} / z 343>245)$ and $\operatorname{RvD} 1(m / z 375>215)$. Identification was matching retention time and diagnostic ions to synthetic standards. ${ }^{43}$ Quantification was performed using standard calibration curves for each, and recoveries were calculated using deuterated internal standard $\left(\mathrm{d}_{4}-\mathrm{PGE}_{2}\right)$.

Histology and immunohistochemistry. After dissection, lungs were perfusion-fixed at $20 \mathrm{cmH}_{2} \mathrm{O}$ in IHC zinc fixative buffer (BD Pharmingen, San Diego, CA). Tissue blocks were obtained from mid-sagittal slices of lungs embedded in paraffin for staining with hematoxylin and eosin (Sigma). For Ly-6G (1/100 dilution; BD Pharmingen) immunostaining, tissue sections were de-paraffinized and incubated for $1 \mathrm{~h}$ at room temperature with anti-mouse Ly-6G antibody to stain for PMNs. For ALX/FPR2 (1/50 dilution; Santa Cruz Biotechnology) and phosphorylated $\mathrm{p} 65$ subunit of NF- $\mathrm{KB}$ (phosphoS276, 1/50 dilution; Abcam) immunostaining, tissue sections were incubated overnight at $4^{\circ} \mathrm{C}$.

Determination of barrier integrity. FITC-dextran was utilized as a marker of epithelial barrier disruption (as in ref. 42 with minor modification). Briefly, FITC-dextran ( $50 \mathrm{mg} \mathrm{kg}^{-1}$ mouse) was given intratracheally via a $24-\mathrm{G}$ angiocatheter. BAL with $1 \mathrm{ml}$ phosphate-buffered saline was performed $10 \mathrm{~min}$ later with concurrent collection of blood from the right ventricle. BAL and serum were loaded onto a microwell plate and fluorescence was determined (excitation, $490 \mathrm{~nm}$; emission, $520 \mathrm{~nm}$ ). The ratio of serum to BALF fluorescence was used as an index of airway epithelial permeability. To measure vascular permeability, Evans blue dye was utilized as a marker for endothelial barrier disruption (as in ref. 8). Briefly, Evans blue dye $\left(40 \mathrm{mg} \mathrm{kg}^{-1}\right)$ was injected via tail vein and $30 \mathrm{~min}$ later BAL was performed with $1 \mathrm{ml}$ phosphate-buffered saline. Extravasation of dye into BALFs was quantified by spectrophotometry (absorbance at $650 \mathrm{~nm}$ ).

Measurement of lung mechanics. For measurement of tissue elastance and lung resistance, mice were anesthetized with pentobarbital $\left(70 \mathrm{mg} \mathrm{kg}^{-1}\right.$, intraperitoneally; Abbott Laboratories, North Chicago, IL) and mechanically ventilated with a flexiVent small animal ventilator (SCIREQ, Montreal, QC, Canada). Lung mechanics were determined in anesthetized, ventilated animals.

Mediator levels in BALFs. Select cytokines, chemokines, and total tumor growth factor- $\beta 1$ were measured in aliquots of BALF by cytokine bead array (Aushon Biosystems, Billerica, MA). BALF epinephrine, leukotriene $\mathrm{B}_{4}, 8$-isoprostane, IL-10, and $\mathrm{LXA}_{4}$ levels were measured with specific enzyme-linked immunosorbent assays as per the manufacturer's instructions (epinephrine, Rocky Mountain Diagnostics, Colorado Springs, CO; leukotriene $\mathrm{B}_{4}, 8$-isoprostane, Cayman Chemical, Ann Arbor, MI; IL-10, eBioscience; LXA 4 , Neogen, Lexington, KY).

Statistical analysis. Data are expressed as the mean value \pm s.e.m., unless otherwise indicated. Analysis of variance was used to determine significance for differences between more than two groups. For analyses between two groups, cohorts were compared by Mann-Whitney $U$-test. Significance was determined with $P$-values $\leqslant 0.05$. Statistics were performed using GraphPad Prism 5 for Windows (San Diego, CA).

\section{ACKNOWLEDGMENTS}

We thank GuangLi Zhu and Bonna Ith for technical assistance. This research was supported in part by the US National Institutes of Health grants HL068669, Al068084, and P01-GM095467, and postdoctoral fellowships from the American Lung Association and German Society of Pediatric Pulmonology. The content is solely the responsibility of the authors and does not necessarily reflect the official views of NHLBI, NIAID, NIGMS, or the National Institutes of Health.

\section{DISCLOSURE}

$\mathrm{BDL}$ is an inventor on patents assigned to Brigham and Women's Hospital and Partners HealthCare on the uses of anti-inflammatory and pro-resolving lipid mediators. The resolvins used in this study are licensed to Resolvyx for clinical development. BDL has an equity interest in Resolvyx and receives a share of licensing income through Brigham and Women's Hospital. The other authors declared no potential conflict of interest.

\section{(C) 2013 Society for Mucosal Immunology}

\section{REFERENCES}

1. Ware, L.B. \& Matthay, M.A. The acute respiratory distress syndrome. N. Engl. J. Med. 342, 1334-1349 (2000).

2. Rubenfeld, G.D. et al. Incidence and outcomes of acute lung injury. N. Engl. J. Med. 353, 1685-1693 (2005).

3. Bernard, G.R. et al. The American-European Consensus Conference on ARDS. Definitions, mechanisms, relevant outcomes, and clinical trial coordination. Am. J. Respir. Crit. Care Med. 149, 818-824 (1994).

4. Wheeler, A.P. \& Bernard, G.R. Acute lung injury and the acute respiratory distress syndrome: a clinical review. Lancet 369, 1553-1564 (2007).

5. Marik, P.E. Aspiration pneumonitis and aspiration pneumonia. N. Engl. J. Med. 344, 665-671 (2001)

6. Serhan, C.N. Preventing injury from within, using selective cPLA2 inhibitors. Nat. Immunol. 1, 13-15 (2000).

7. Serhan, C.N. Resolution phase of inflammation: novel endogenous antiinflammatory and proresolving lipid mediators and pathways. Annu. Rev. Immunol. 25, 101-137 (2007).

8. Hilberath, J.N. et al. Resolution of Toll-like receptor 4-mediated acute lung injury is linked to eicosanoids and suppressor of cytokine signaling 3 . FASEB J. 25, 1827-1835 (2011).

9. Gadek, J.E. et al. Effect of enteral feeding with eicosapentaenoic acid, gamma-linolenic acid, and antioxidants in patients with acute respiratory distress syndrome. Enteral Nutrition in ARDS Study Group. Crit. Care Med. 27, 1409-1420 (1999)

10. Pacht, E.R. et al. Enteral nutrition with eicosapentaenoic acid, gammalinolenic acid, and antioxidants reduces alveolar inflammatory mediators and protein influx in patients with acute respiratory distress syndrome. Crit. Care Med. 31, 491-500 (2003).

11. Singer, P. et al. Benefit of an enteral diet enriched with eicosapentaenoic acid and gamma-linolenic acid in ventilated patients with acute lung injury. Crit. Care Med. 34, 1033-1038 (2006)

12. Freedman, S.D. et al. Association of cystic fibrosis with abnormalities in fatty acid metabolism. N. Engl. J. Med. 350, 560-569 (2004).

13. Serhan, C.N. et al. Resolvins: a family of bioactive products of omega-3 fatty acid transformation circuits initiated by aspirin treatment that counter proinflammation signals. J. Exp. Med. 196, 1025-1037 (2002).

14. Sun, Y.P. et al. Resolvin D1 and its aspirin-triggered 17R epimer. Stereochemical assignments, anti-inflammatory properties, and enzymatic inactivation. J. Biol. Chem. 282, 9323-9334 (2007).

15. Fukunaga, K., Kohli, P., Bonnans, C., Fredenburgh, L.E. \& Levy, B.D. Cyclooxygenase 2 plays a pivotal role in the resolution of acute lung injury. J. Immunol. 174, 5033-5039 (2005)

16. O'Neal, H.R. et al. Prehospital statin use is associated with a lower incidence of sepsis and ALI. Am. J. Respir. Crit. Care Med. 179, A1155 (2009).

17. Krishnamoorthy, S. et al. Resolvin D1 binds human phagocytes with evidence for proresolving receptors. Proc. Natl. Acad. Sci. USA 107, 1660-1665 (2010)

18. Krishnamoorthy, S., Recchiuti, A., Chiang, N., Fredman, G. \& Serhan, C.N. Resolvin D1 receptor stereoselectivity and regulation of inflammation and proresolving microRNAs. Am. J. Pathol. 180, 2018-2027 (2012).

19. Zarbock, A., Singbartl, K. \& Ley, K. Complete reversal of acid-induced acute lung injury by blocking of platelet-neutrophil aggregation. J. Clin. Invest. 116, 3211-3219 (2006)

20. Looney, M.R. et al. Platelet depletion and aspirin treatment protect mice in a two-event model of transfusion-related acute lung injury. J. Clin. Invest. 119, 3450-3461 (2009). 
21. Bonnans, C.. Fukunaga, K., Levy, M.A. \& Levy, B.D. Lipoxin A(4) regulates bronchial epithelial cell responses to acid injury. Am. J. Pathol. 168, 1064-1072 (2006).

22. Chiang, N. et al. The lipoxin receptor ALX: potent ligand-specific and stereoselective actions in vivo. Pharmacol. Rev. 58, 463-487 (2006).

23. Norling, L.V., Dalli, J., Flower, R.J., Serhan, C.N. \& Perretti, M. Resolvin D1 limits PMN recruitment to inflammatory loci: receptor dependent actions. Arterioscler. Thromb. Vasc. Biol. advance online publication, 12 April 2012 (e-pub ahead of print); PMID: 22499990 (2012).

24. Claria, J. \& Serhan, C.N. Aspirin triggers previously undescribed bioactive eicosanoids by human endothelial cell-leukocyte interactions. Proc. Natl. Acad. Sci. USA 92, 9475-9479 (1995).

25. Serhan, C.N., Chiang, N. \& Van Dyke, T.E. Resolving inflammation: dual anti-inflammatory and pro-resolution lipid mediators. Nat. Rev. Immunol. 8, 349-361 (2008).

26. Bento, A.F., Claudino, R.F., Dutra, R.C., Marcon, R. \& Calixto, J.B. Omega-3 fatty acid-derived mediators $17(R)$-hydroxy docosahexaenoic acid, aspirin-triggered resolvin D1 and resolvin D2 prevent experimental colitis in mice. J. Immunol. 187, 1957-1969 (2011).

27. Lima-Garcia, J.F. et al. The precursor of resolvin D series and aspirintriggered resolvin D1 display anti-hyperalgesic properties in adjuvantinduced arthritis in rats. Br. J. Pharmacol. 164, 278-293 (2011).

28. Abraham, E. Neutrophils and acute lung injury. Crit. Care Med. 31, S195-S199 (2003).

29. Knight, P.R., Druskovich, G., Tait, A.R. \& Johnson, K.J. The role of neutrophils, oxidants, and proteases in the pathogenesis of acid pulmonary injury. Anesthesiology 77, 772-778 (1992).

30. Folkesson, H.G., Matthay, M.A., Hebert, C.A. \& Broaddus, V.C. Acid aspiration-induced lung injury in rabbits is mediated by interleukin-8dependent mechanisms. J. Clin. Invest. 96, 107-116 (1995).

31. Kasuga, K. et al. Rapid appearance of resolvin precursors in inflammatory exudates: novel mechanisms in resolution. J. Immunol. 181, 8677-8687 (2008).
32. Wang, B. et al. Resolvin D1 protects mice from LPS-induced acute lung injury. Pulm. Pharmacol. Ther. 24, 434-441 (2011).

33. Dartt, D.A. et al. Conjunctival goblet cell secretion stimulated by leukotrienes is reduced by resolvins D1 and $\mathrm{E} 1$ to promote resolution of inflammation. J. Immunol. 186, 4455-4466 (2011).

34. Chiang, N. et al. Infection regulates pro-resolving mediators that lower antibiotic requirements. Nature 484, 524-528 (2012).

35. Levy, B.D., Clish, C.B., Schmidt, B., Gronert, K. \& Serhan, C.N. Lipid mediator class switching during acute inflammation: signals in resolution. Nat. Immunol. 2, 612-619 (2001).

36. Schwab, J.M., Chiang, N., Arita, M. \& Serhan, C.N. Resolvin E1 and protectin D1 activate inflammation-resolution programmes. Nature 447 , 869-874 (2007).

37. Frenette, P.S. et al. Platelet-endothelial interactions in inflamed mesenteric venules. Blood 91, 1318-1324 (1998).

38. Zarbock, A. \& Ley, K. The role of platelets in acute lung injury (ALI). Front. Biosci. 14, 150-158 (2009).

39. Fredman, G., Van Dyke, T.E. \& Serhan, C.N. Resolvin E1 regulates adenosine diphosphate activation of human platelets. Arterioscler. Thromb. Vasc. Biol. 30, 2005-2013 (2010).

40. Mayer, K. et al. Acute lung injury is reduced in fat- 1 mice endogenously synthesizing $n-3$ fatty acids. Am. J. Respir. Crit. Care Med. 179, 474-483 (2009).

41. Bilal, S. et al. Fat-1 transgenic mice with elevated omega-3 fatty acids are protected from allergic airway responses. Biochim. Biophys. Acta 1812 1164-1169 (2011).

42. Haworth, O., Cernadas, M., Yang, R., Serhan, C.N. \& Levy, B.D. Resolvin E1 regulates interleukin 23 , interferon-gamma and lipoxin A4 to promote the resolution of allergic airway inflammation. Nat. Immunol. 9, 873-879 (2008).

43. Yang, R., Chiang, N., Oh, S.F. \& Serhan, C.N. Metabolomics-lipidomics of eicosanoids and docosanoids generated by phagocytes. Curr Protoc. Immunol. Chapter 14, Unit 14.26 (2011). 